\title{
Barriers and facilitators to discontinuing antidepressant use: a systematic review and thematic synthesis
}

Emma Maund, Rachel Dewar-Haggart, Samantha Williams, Hannah Bowers, Adam W A Geraghty, Geraldine Leydon, Carl May, Sarah Dawson, Tony Kendrick

University of Southampton Primary Care \& Population Sciences, Aldermoor Health Centre, Southampton SO16 5ST, UK etmaund@gmail.com Emma Maund Research Fellow

University of Southampton Primary Care \& Population Sciences, Aldermoor Health Centre, Southampton SO16 5ST, UK R.V.Dewar-Haggart@soton.ac.uk Rachel Dewar-Haggart Postgraduate student

University of Southampton Primary Care \& Population Sciences, Aldermoor Health Centre, Southampton SO16 5ST, UK s.j.williams@soton.ac.uk Samantha Williams Qualitative Senior Research Assistant

University of Southampton Primary Care \& Population Sciences, Aldermoor Health Centre, Southampton SO16 5ST, UK H.M.Bowers@soton.ac.uk Hannah Bowers Research Fellow

University of Southampton Primary Care \& Population Sciences, Aldermoor Health Centre, Southampton SO16 5ST, UK A.W.Geraghty@soton.ac.uk Adam WA Geraghty Senior Research Fellow 
University of Southampton Primary Care \& Population Sciences, Aldermoor Health Centre, Southampton SO16 5ST, UK gerry@soton.ac.uk Geraldine Leydon Professor of Medical Sociology \& Healthcare Interaction

Department of Health Services Research and Policy, Faculty of Public Health and Policy, London School of Hygiene and Tropical Medicine, 15-17 Tavistock Place, London WC1H 9SH, UK. carl.may@Ishtm.ac.uk Carl May Professor of Medical Sociology

Department of Population Health Sciences, Bristol Medical School, University of Bristol, Canynge Hall, Bristol, BS8 2PS, UK sarah.dawson@bristol.ac.uk Sarah Dawson Senior Research Associate in Information Retrieval

University of Southampton Primary Care \& Population Sciences, Aldermoor Health Centre, Southampton SO16 5ST ark1@soton.ac.uk Tony Kendrick Professor of Primary Care Correspondence to: ark1@soton.ac.uk Tony Kendrick Professor of Primary Care

Word count: 4785 


\section{ABSTRACT}

\section{Objective}

To explore patient and health professional views and experiences of antidepressant treatment with particular focus on barriers and facilitators to discontinuing use.

\section{Design}

Systematic review with thematic synthesis

\section{Data sources}

MEDLINE, PubMed, Embase, PsycINFO, CINAHL, AMED, Health Management Information Consortium, OpenGrey, and the Networked Digital Library of Theses and Dissertations from inception until February 2017. Updated searches were carried out in July 2018.

\section{Eligibility criteria}

Primary studies, published in English, that used qualitative data collection and analysis, and had data on attitudes, beliefs, feelings, perceptions on continuing or discontinuing antidepressant use, of patients (aged 18 or above, who received treatment with antidepressants for at least 6 months) or any health professionals.

\section{Data extraction}

One reviewer extracted data and assessed study quality, which was checked by a second reviewer.

\section{Findings}

Twenty two papers were included in the review. A thematic synthesis was performed for patient perspectives only, due to insufficient data from a health professional perspective. The thematic synthesis yielded nine themes: (1) psychological and physical capabilities; (2) perception of antidepressants; (3) fears; (4) intrinsic motivators and goals; (5) the Doctor as a navigator to maintenance or discontinuation; (6) perceived cause of depression; (7) aspects of information that support decision-making; (8) significant others - a help or a hindrance; and (9) support from other health professionals.

\section{Limitations}

Coding and development of subthemes and themes was performed by one researcher and further developed through discussion between two researchers. 


\section{Conclusions}

Barriers and facilitators to discontinuing antidepressant use are numerous and complex, and likely to require detailed conversations between patients and their general practitioners (GPs). These conversations are more likely to happen if GPs raise the issue of discontinuation. Further research from a health professional perspective including, but not limited to GPs, is needed.

Keywords

Depression; antidepressants; primary care; discontinuation; qualitative; systematic review

Systematic review registration

PROSPERO 2016 CRD42016053941 


\section{INTRODUCTION}

In Western countries, antidepressant prescriptions have risen steadily over time (McCarthy, 2013). In England, in 2016 there were over 64 million prescriptions, more than twice as many compared to 2006, at a cost of $£ 267$ million (Health and Social Care Information Centre, 2017). The main reason for this increase is increasing long-term use, with a median length of treatment now greater than two years (Kendrick et al., 2015; McCrea et al., 2016; Moore et al., 2009). While some people need to use antidepressants long-term to prevent relapse, studies suggest that $30-50 \%$ of long-term users have no evidence-based indication to continue and could try stopping (Cruickshank et al., 2008; Ambresin et al., 2015; Piek et al., 2014).

Continuation of antidepressants exposes patients to potential side effects including sleep disturbance, weight gain, sexual dysfunction and gastrointestinal bleeding (Ferguson et al., 2001). In the elderly, antidepressant use is associated with increased risk of strokes, falls, fractures, hyponatraemia and death (Coupland et al., 2011). It also results in substantial healthcare costs (Health and Social Care Information Centre, 2017; Vasiliadis et al., 2013).

The REDUCE programme (Kendrick, 2016), in which all but one review authors are involved, aims to develop safe and effective support for discontinuing long-term antidepressant use where appropriate. The act of stopping any long-term medication is complicated by many factors related to patients and prescribers (Reeve et al., 2013; Anderson et al., 2014). These need to be understood to develop safe and effective approaches to stopping medication. We therefore performed a scoping search to identify key qualitative literature on factors influencing patients' and prescribers' decisions to discontinue long-term antidepressants. We found three relevant reviews: a 2009 metaethnography of patient experiences of antidepressants, including stopping treatment (Malpass et al., 2009); and two thematic synthesis, one from the patient perspective and one from the prescriber's, of barriers and facilitators to stopping any long-term medication (Reeve et al., 2013; Anderson et al., 
2014). All three reviews included studies relating to antidepressant discontinuation. However, we also identified relevant primary qualitative studies that were not included in any of these reviews. We therefore conducted a systematic review of qualitative studies exploring patient and health professional views and experiences of antidepressant treatment with particular focus on barriers and facilitators to discontinuing use.

\section{METHODS}

\section{Protocol and registration}

The review protocol was registered with the PROSPERO International Prospective Register of Systematic Reviews

(http://www.crd.york.ac.uk/PROSPERO/display_record.php?ID=CRD42016053941)

\section{Eligibility criteria}

We included primary studies that: (1) used qualitative data collection (e.g. interviews, focus groups) and analysis, including qualitative components of mixed methods studies, and (2) had data, regardless of study aims, on attitudes, beliefs, feelings, perceptions on continuing or discontinuing antidepressant use, of patients (aged 18 or above, who received treatment with antidepressants for at least 6 months) or health care professionals with particular focus on barriers and facilitators to discontinuation. Although we did not restrict by study aims, it needed to be clear from the abstract that there was relevant information for the aims of our research. Furthermore, we did not restrict type of health professional (e.g. general practitioners, nurses, psychiatrists, pharmacists, primary care mental health workers, psychotherapists, nursing home staff) or setting.

We excluded studies that: (1) used survey data, including open responses to survey questions, (2) defined continuation or discontinuation of treatment in terms of adherence or non-adherence in the initial stage of treatment only (less than 6 months), or (3) were not published in English.

\section{Search strategy and sources}

We searched the following databases from inception until 6 February 2017: MEDLINE (Ovid), PubMed, Embase (Ovid), PsycINFO (EBSCOhost), CINAHL (EBSCOhost), AMED (EBSCOhost), Health 
Management Information Consortium, OpenGrey, and the Networked Digital Library of Theses and Dissertations from inception. Updated searches were undertaken in July 2018.

The MEDLINE search strategy (appendix 1) was developed by an experienced health librarian (SD). The strategy consisted of terms and text words of antidepressants and discontinuation, and a modified qualitative research filter (DeJean et al., 2016). This strategy was then adapted by one researcher (EM) for the remaining databases.

We also performed citation searching, reference list checking and related article checking for full papers meeting the inclusion criteria from our initial database searches, and contacted experts in the field. Related article searching was performed using the "related articles" feature in PubMed, and stopped after 100 suggestions for resource limitations.

\section{Study selection}

One researcher (EM) screened all titles and abstracts yielded by searches against inclusion criteria. Two researchers (TK and RR) screened a 10\% sample of titles and abstracts each. We obtained full papers for all titles and abstracts appearing to meet inclusion criteria or where there was uncertainty. Two researchers (EM and TK) then independently assessed whether these full papers met the inclusion criteria. Any disagreements were resolved by discussion.

\section{Data collection/extraction process}

Data extraction was performed into a standardised form created in Excel by one researcher (EM) and checked by two researchers (HB and TK). Data extracted included: research setting, aims of study, theoretical background, sampling approach, participant characteristics, data collection methods, data analysis approach, and findings.

\section{Assessment of study quality}

There is debate over what criteria to use to assess study quality when conducting qualitative evidence syntheses, therefore we used two different methods (Carroll et al., 2015) 
Each study was assessed against the CASP criteria for qualitative research (Critical Appraisal Skills Programme, 2017). This checklist supports critical appraisal of research by asking reviewers questions about 10 domains that should be considered in qualitative research. Prior experience within the review group found the consolidated criteria for reporting qualitative research (COREQ) 32-item checklist allowed a deeper assessment of study quality than other tools (Tong et al., 2007). We therefore also used COREQ in this review. Assessment of study quality was performed by one reviewer (EM) and checked by two reviewers (HB and TK), with any disagreements resolved by discussion. A priori we decided not to exclude studies from the review or analysis based on their assessed quality (Carroll et al., 2015). Post-hoc, we performed sensitivity analyses of the effects of study quality on our findings, by excluding studies with poorer assessments, compared to other studies, in any CASP domain.

\section{Data synthesis}

We conducted a thematic synthesis to synthesise findings (Thomas and Hardsen, 2008). All included papers were imported into NVivo (version 11). Following multiple readings to achieve immersion, one researcher (EM) performed line by line coding of participants' views and authors' interpretation of their study findings that touched on continuing or discontinuing antidepressant use, regardless of where they were located in the paper. Papers were coded in order of publication, beginning with the oldest. Codes, sub-themes and themes were developed iteratively as papers were analysed. Sub-themes and themes were further developed and refined through discussion between two researchers (EM and AG). Sensitivity analyses were performed by excluding studies with special populations e.g. those with experience of treatment for illicit drug use.

At monthly meetings we discussed the research process, it's progress, and our findings with our Public Involvement (PPI) colleagues. 


\section{FINDINGS}

\section{Study selection}

The initial search yielded 4710 records in total, including 3455 unique records (figure 1). Of these 3373 were ineligible after review of title and abstract. Of 82 remaining papers, one was unobtainable, and 60 were excluded after assessment of full papers (abbreviated reports of full papers included in the review $n=2$; perspective was neither adult patients nor healthcare professionals $n=4$; did not use qualitative data collection and analysis $n=23$; no data on attitudes, beliefs, feelings, perceptions on continuing or discontinuing antidepressant use $\mathrm{n}=31$ ). Twenty one papers, reporting 21 studies, were therefore included after the initial search. The updated search yielded 912 records, of which 664 were unique. Of these, 661 were ineligible after reviews of titles and abstracts. Of the three full papers assessed, two were excluded (no data on attitudes, beliefs, feelings, perceptions on continuing or discontinuing antidepressant use $n=1$, did not use qualitative data collection and analysis $\mathrm{n}=1$ ). A total of 22 papers reporting 22 studies were therefore included in our review (Karp, 1993; Knudsen et al., 2002; Pollack and Grime, 2002; Hoogen, 2006; Verbeek-Heida and Mathot, 2006; Holt, 2007; Johnston et al., 2007; Leydon et al., 2007; Wilson, 2007; McMullen and Herman, 2009; Aselton, 2010; Dickinson et al., 2010; McKinney and Greenfield, 2010; Iden et al., 2011; Schofield et al., 2011; Buus et al., 2012; Bayliss and Holttum 2015; Eveleigh, 2015; Nygaard et al., 2015; Weaver, 2015; Bosman et al., 2016; Johnson et al., 2017). Of note, no potentially relevant titles and abstracts were excluded solely on the basis of being non-English language publications.

\section{Study characteristics}

Characteristics of included studies are presented in appendix 2. Studies dated from 1993-2017 and were carried out in the UK, Netherlands, Denmark, Norway, USA, Canada, Australia and New Zealand. One study was of general practitioner (GP) perspectives only, one of GPs and nurses working in nursing homes, four studies were of general practitioner (GP) and patient perspectives (including one of patients aged 75 and over), and 16 from patient perspectives only. Of these 16 
studies, 11 were in specific populations (women only, including one of pregnant women only, $n=5$; aged 19-24, $n=2$; participants in experiments to support antidepressant discontinuation, $n=2$; experience of treatment for illicit drug use, $n=1$; experience of using antidepressants and CBT, $n=1$ ). Sixteen studies were reported in journal articles, three in PhD theses, one in a Doctor of Clinical Psychology thesis, one in an MA thesis, and one as a final report of a research fellowship. Where reported, minimum duration of antidepressant use ranged from 6 months to 1 year, and maximum duration from 4 years to greater than 19 years.

\section{Quality assessment}

Assessed against the CASP criteria (appendix 3), all studies had a clear statement of aims for which qualitative methodology was appropriate, and all were considered valuable research.

Assessed against the 32 item COREQ checklist, all items were reported by a minimum of two studies (appendix 4). All 22 studies reported sample size, description of sample, use of interview guide, participant quotations, and had consistency between data and reported findings. Twenty one studies reported on the methodological theory that underpinned their study, the sampling method used, and had clear major themes or minor themes. Only two studies, both PhD theses, reported a description of their coding tree.

\section{Synthesis of results}

Data from all studies were initially coded. Data from all studies were initially coded, with data from 21 studies found in the abstract $(n=1)$, results $(n=21)$, or discussion $(n=11)$. A further study was reported in a Master of Arts thesis, which did not employ a typical IMRaD (Introduction, Methods, Results and Discussion) format (Hoogen, 2006). Quotations from participants were found throughout this document. Due to the limited number of studies and "thinness" of the data, there was insufficient data to perform a meaningful thematic synthesis for health professional perspectives. The thematic synthesis presented in the following is therefore from patient perspectives only. Sub-themes were however cross-checked with coded data 
from the healthcare professionals' perspective. For codes assigned to more than one study, concordance or discordance with sub-themes is highlighted below.

Thematic synthesis yielded nine themes, and 49 sub-themes (see figure 2 and appendix 5). These themes are listed below, in decreasing order of the quantity of coded data contained within them:

1. Psychological and physical capabilities

2. Perception of antidepressants

3. Fears

4. Intrinsic motivators and goals

5. The Doctor as a navigator to maintenance or discontinuation

6. Perceived cause of depression

7. Aspects of information that support decision-making

8. Significant others - a help or a hindrance

9. Support from other health professionals

\section{Psychological and physical capabilities}

This theme refers to patients' beliefs about their capabilities to discontinue antidepressants, the possession of effective coping skills e.g. mindfulness, knowledge and experience of antidepressant discontinuation, perceived dependence on antidepressants, intermittent need for antidepressants, and stability of life circumstances. This theme was mentioned in 17 studies (Karp, 1993; Knudsen et al., 2002; Hoogen, 2006; Verbeek-Heida and Mathot, 2006; Holt, 2007; Leydon et al., 2007; Wilson, 2007; McMullen and Herman, 2009; Aselton, 2010; Dickinson et al., 2010; McKinney and Greenfield, 2010; Schofield et al., 2011; Buus et al., 2012; Bayliss and Holttum 2015; Eveleigh, 2015; Weaver, 2015; Bosman et al., 2016).

There were two main barriers to discontinuation. The first was a patient's dependence (psychological, physical or undefined) on antidepressants, which was described in 10 studies (Karp, 
1993; Knudsen et al., 2002; Verbeek-Heida and Mathot, 2006; Wilson, 2007; Dickinson et al., 2010; McKinney and Greenfield, 2010; Schofield et al., 2011; Bayliss and Holttum 2015; Eveleigh, 2015; Weaver, 2015), e.g.:

[Patient:] "It's psychological that dependence. You're afraid everything will go wrong if you don't take your medicine" (Knudsen et al., 2002)

The second was problematic experiences (self or others) of discontinuing antidepressants, which was described in nine studies (Verbeek-Heida and Mathot, 2006; Leydon et al., 2007; Wilson, 2007; Dickinson et al., 2010; Schofield et al., 2011; Buus et al., 2012; Bayliss and Holttum 2015; Eveleigh, 2015; Bosman et al., 2016), e.g.:

[Patient:] "I have tried to stop, I did foolishly, foolishly try to stop and I just stopped taking them. That was a mistake, big mistake." (Leydon et al., 2007)

The three main facilitators were: confidence in capability to discontinue (Karp, 1993; McMullen and Herman, 2009; Eveleigh, 2015; Weaver, 2015), effective coping strategies (Wilson, 2007; Bayliss and Holttum 2015; Weaver, 2015), and stable life circumstances (McMullen and Herman, 2009; McKinney and Greenfield, 2010; Bosman et al., 2016). Confidence in capability to discontinue and effective coping strategies were often interlinked. For example, in a study of patients who had received mindfulness-based cognitive course to help discontinue antidepressants, one patient stated:

"My take on it was, well, there's the pills, there's the, um, meditation and the processes I've learnt. So, it- it gave me confidence I think.. That I had, um, I had a way of dealing with it...not just come off the pills... but it gave me confidence that I could cope." (Weaver, 2015) 
From a GP perspective, patients' psychological dependence (Pollack and Grime, 2002; Bosman et al., 2016; Johnson et al., 2017), and difficult life circumstances (Pollack and Grime, 2002; Dickinson et al., 2010; Bosman et al., 2016), were also barriers to discontinuation.

\section{Perception of antidepressants}

This encompasses patients' characterisation of antidepressants (natural/benign versus negative/unnatural characterisation), their effect (positive, lack of, or negative), and feelings about long term antidepressant use, and these were evident in 16 studies (Karp, 1993; Pollack and Grime, 2002; Hoogen, 2006; Verbeek-Heida and Mathot, 2006; Holt, 2007; Leydon et al., 2007; Wilson, 2007; McMullen and Herman, 2009; Aselton, 2010; Dickinson et al., 2010; McKinney and Greenfield, 2010; Schofield et al., 2011; Buus et al., 2012; Bayliss and Holttum 2015; Eveleigh, 2015; Bosman et al., 2016). A perceived positive effect of antidepressant use appeared to be the main barrier to discontinuation (Karp, 1993; Hoogen, 2006; Verbeek-Heida and Mathot, 2006; Dickinson et al., 2010; McKinney and Greenfield, 2010; Eveleigh, 2015; Bosman et al., 2016), e.g.:

[Patient:] "Well, I am feeling very well, I am very stable. I'm in harmony, I don't have any mood swings or anything. I don't think I could feel any better than I do now. Also mentally. So, I won't risk it. I won't attempt it, maybe it would be successful, but I won't dare to try." (Eveleigh, 2015)

Experiencing unacceptable side effects was the main facilitator to discontinuation (Karp, 1993; Holt, 2007; Wilson, 2007; McMullen and Herman, 2009; Aselton, 2010; Schofield et al., 2011; Buus et al., 2012; Bayliss and Holttum 2015; Bosman et al., 2016). These included both physical and psychiatric side effects, with the most frequent being emotional numbing, e.g.:

[Patient:] "I absolutely hated taking antidepressants. I think they mute people. It makes you a blank human being who is unable to fully express emotions. I would rather have some crazy ups and downs than be static. This is why I stopped taking antidepressants. I've actually been fine and it's been about four months." (Aselton, 2010) 
This specific side effect was mentioned as a reason to discontinue antidepressants in five studies, including one in patients currently or recently undergoing treatment for illicit drug use (Holt, 2007; Wilson, 2007; Aselton, 2010; Schofield et al., 2011; Bayliss and Holttum 2015). However, in one study this side effect was described in terms of a positive effect, thereby serving as a barrier to discontinuation:

[Study author:] "Antidepressants had a greater role to play in making un-problematic the difficult relationships in Kylie's life. The numbing effects of the drug protected Kylie from some of the pain she was used to experiencing in interactions with her family, especially her parents, allowing her to respond differently to those provocations in the interest of learning to have a different kind of relationship. This seemed only somewhat successful in interactions with her parents-Kylie reported that their hurtful patterns of relating to her remained unchanged; though, once she was back on the antidepressants, she could ignore them better." (Hoogen, 2006)

Interestingly, for older patients ( $\geq 75$ years), there was a noted lack of concern from both themselves and their GPs over side effects:

[Patient:] "Neither patients nor GPs had concerns about side effects and this provides little apprehension in the initiation and maintenance of antidepressants" (Dickinson et al., 2010)

\section{Fears}

Fears relating to stopping or continuing antidepressants were mentioned in 15 studies (Karp, 1993; Knudsen et al., 2002; Pollack and Grime, 2002; Hoogen, 2006; Verbeek-Heida and Mathot, 2006; Leydon et al., 2007; McMullen and Herman, 2009; Dickinson et al., 2010; Schofield et al., 2011; Buus et al., 2012; Bayliss and Holttum 2015; Eveleigh, 2015; Nygaard et al., 2015; Weaver, 2015; Bosman et al., 2016). 
The overriding fear that served as a barrier to discontinuation, and the most frequently used code in this study, was of relapse (Karp, 1993; Knudsen et al., 2002; Pollack and Grime, 2002; Hoogen, 2006; Verbeek-Heida and Mathot, 2006; Leydon et al., 2007; Schofield et al., 2011; Buus et al., 2012; Bayliss and Holttum 2015; Eveleigh, 2015; Nygaard et al., 2015; Weaver, 2015; Bosman et al., 2016), e.g.:

[Patient:] "I don't dare to stop, the fear that all will come back as it was before" (Verbeek-Heida and Mathot, 2006)

GPs were also fearful of causing relapse due to reducing or stopping antidepressants (Bosman et al., 2016; Johnson et al., 2017).

Fear of withdrawal effects was another patient barrier to discontinuation (Leydon et al., 2007; Dickinson et al., 2010; Schofield et al., 2011; Bayliss and Holttum 2015; Eveleigh, 2015; Bosman et al., 2016), e.g.:

"LR [interviewer]: Is there anything else that ..... makes you keep taking the drugs?

$P$ [patient]: ... the major factor is the side effects of coming off them .... I don't think I take them to sustain my mood but purely just to stop the side effects" (Leydon et al., 2007)

In contrast, fear of addiction was the main facilitator to discontinuation (Verbeek-Heida and Mathot, 2006; McMullen and Herman, 2009; Schofield et al., 2011; Bayliss and Holttum 2015; Eveleigh, 2015; Bosman et al., 2016), e.g.:

"JH [interviewer]: two years um so what kinds of things what led you to decide to come off of antidepressants.

Veronica [patient]: Um I guess some of it about it in the media and um worrying about becoming 
addicted“ (McMullen and Herman, 2009)

\section{Intrinsic motivators and goals}

This refers to factors that arise entirely from within an individual in order to satisfy basic psychological needs for autonomy, competence, and relatedness (Deci and Ryan, 2002). Aspects include self-identity, self-stigma, goal priority, irrationality, and desire to function independently of antidepressants. This theme was described in 14 studies (Karp, 1993; Knudsen et al., 2002; Hoogen, 2006; Verbeek-Heida and Mathot, 2006; Leydon et al., 2007; McMullen and Herman, 2009; Aselton, 2010; Dickinson et al., 2010; McKinney and Greenfield, 2010; Schofield et al., 2011; Buus et al., 2012; Eveleigh, 2015; Nygaard et al., 2015; Bosman et al., 2016).

Self-identity was both a barrier and facilitator to discontinuation. A self-identity of "old", and "disabled" were barriers to discontinuation (Hoogen, 2006; Dickinson et al., 2010; Schofield et al., 2011), while a self-identity of "healthy" and "true-self" were facilitators (Karp, 1993; Knudsen et al., 2002; Verbeek-Heida and Mathot, 2006; Leydon et al., 2007; Aselton, 2010; Schofield et al., 2011; Bosman et al., 2016).

The self-identity of being "a good mother" was found to be both a barrier and a facilitator (Hoogen, 2006; McMullen and Herman, 2009; Nygaard et al., 2015),e.g.:

[Patient:] "I really feel like I can do it right this time-that, if I take the antidepressants, I can be the kind of mommy I need to be." (Hoogen, 2006) [Patient:] "I had jitters and other um problem uh really with sleeping just about right around the clock trying to be a mom but I couldn't. And my daughter hearing my daughter say you're not like my mom. You're not my mom any more. You're always sleeping. You never wanna go out with me. You don't wanna go to the park. You want to do nothing. And that really was sad for me. Made me [mhm]. So I just said that's it. I will never touch another med again. So I quit them altogether." (McMullen and Herman, 2009) 


\section{The Doctor as a navigator to maintenance or discontinuation of antidepressants}

This theme refers to the role of the Doctor in directing the patient towards maintaining or discontinuing antidepressants. It encompasses the Doctor's recommendation/approval to continue or discontinue antidepressants, their role in initiating discussions about discontinuation with the patient, support/guidance of the discontinuation process, work practices e.g. lack of review, and work issues e.g. lack of time. This theme was mentioned in 13 studies (Karp, 1993; Pollack and Grime, 2002; Verbeek-Heida and Mathot, 2006; Johnston et al., 2007; Leydon et al., 2007; Wilson, 2007; Dickinson et al., 2010; McKinney and Greenfield, 2010; Schofield et al., 2011; Bayliss and Holttum 2015; Eveleigh, 2015; Nygaard et al., 2015; Bosman et al., 2016). In most cases the Doctor referred to was a GP.

A key facilitator was the patient's doctor recommending or approving the discontinuation of antidepressants (Pollack and Grime, 2002; Verbeek-Heida and Mathot, 2006; Johnston et al., 2007; Eveleigh, 2015; Nygaard et al., 2015), e.g.:

[Patient:] "...if you get the advice, that it should be possible, then you start to think, maybe I should try. Because you do want to live without. It gave me the extra nudge that I needed to give it a try" (Eveleigh, 2015)

In contrast, there were two key barriers to discontinuation. The first was lack or inadequacy of doctor support (Verbeek-Heida and Mathot, 2006; Eveleigh, 2015; Bosman et al., 2016), e.g.:

Patient:] "I think that the GP knows a lot, but only little bits. They only have basic knowledge about many areas. I do not blame them for that, but I do have more faith in the experts. I think that the GP should refer me sooner to specialised care, because they cannot know everything." (Bosman et al., 2016) 
The second was a belief it was the Doctor's responsibility to initiate discussions about discontinuation (Verbeek-Heida and Mathot, 2006; Dickinson et al., 2010; Bosman et al., 2016), e.g.:

[Patient:] "I do think that the GP is responsible for his patient, and should therefore also take the initiative around antidepressant treatment" (Bosman et al., 2016)

Furthermore, from both a patient and GP perspective, work practices in the form of a lack of GP review (Leydon et al., 2007; Dickinson et al., 2010; Iden et al., 2011; Schofield et al., 2011; Bayliss and Holttum 2015; Bosman et al., 2016; Johnson et al., 2017), and work issues of lack of time (Leydon et al., 2007; Dickinson et al., 2010; Bayliss and Holttum 2015; Bosman et al., 2016), were barriers to discontinuation. For example, in the case of lack of GP review:

"LR [interviewer]: How often would you see your GP about it? P [patient]: I haven't seen him for ... my review date is ... was last November, so I, I, they keep lining up, I must make an appointment to go and see him so that is down to me .... They keep signing the form so I keep on doing it." (Leydon et al., 2007)

GP: 'Sometimes you are just very busy and you think "let's get those repeat prescriptions over and done with" (Bosman et al., 2016)

\section{Perceived cause of depression}

This theme refers to patients' beliefs or opinions about the causes of depression, and therefore the appropriate treatment. The belief that the depression was a long-term condition requiring long term treatment, or had a biochemical cause, was apparent in 10 studies (Karp, 1993; Pollack and Grime, 2002; Hoogen, 2006; Verbeek-Heida and Mathot, 2006; Johnston et al., 2007; Dickinson et al., 2010; Schofield et al., 2011; Buus et al., 2012; Eveleigh, 2015; Bosman et al., 2016), e.g.: 
[Patient:] "I am aware that my body is supplied with substances [the medicine] that my brain does not produce enough of. I get that, and that they are really important substances inside my brain, that do this and that, but I don't know how it gets in and works. I know that it is something I need. And that is ok. I'm fine with that." (Buus et al., 2012)

In contrast, this theme only appeared as a facilitator, in terms of the belief that depression was seasonal or caused by life circumstances, in one paper (McMullen and Herman, 2009).

\section{Aspects of information that support decision-making}

This refers to aspects of information concerning the process, and risk and benefits of discontinuing antidepressants. This theme was addressed in five studies (Pollack and Grime, 2002; Verbeek-Heida and Mathot, 2006; Leydon et al., 2007; Nygaard et al., 2015; Bosman et al., 2016).

Incongruent or insufficient information on discontinuation was a barrier to discontinuation (Verbeek-Heida and Mathot, 2006; Leydon et al., 2007; Nygaard et al., 2015; Bosman et al., 2016), while information on how to discontinue and what to expect was a facilitator, (Pollack and Grime, 2002; Bosman et al., 2016) e.g.:

[Patient:] "I want to come off them and no-one will help me, and I don't know what the effects will be if I come off them myself cause nobody will tell me." (Leydon et al., 2007)

[Patient:] "I've just no idea what it would involve that's why I'm frightened to come off them" (Leydon et al., 2007)

\section{Significant others - a help or a hindrance}

This theme refers to the role that patients' significant others (life partner, family, children, friends) play in encouraging or discouraging patients from discontinuing antidepressants. This theme was addressed in seven studies (Karp, 1993; Hoogen, 2006; McMullen and Herman, 2009; Schofield et al., 2011; Eveleigh, 2015; Nygaard et al., 2015; Bosman et al., 2016). 
Significant others could exert pressure on patients to continue or discontinue antidepressants, e.g.:

[Patient:] "She [my wife] really does understand that I would like to try to taper, it's not like she doesn't want me to. But she said please take the pills, because you're so much easier to handle... ha ha. So I just keep taking them." (Eveleigh, 2015)

[Patient:] "Well my family told me to stop taking them, they weren't happy about it" (Schofield et al., 2011)

Finally, significant others could facilitate discontinuation by providing support and guidance through the discontinuation process e.g. by monitoring patients' symptoms (Nygaard et al., 2015; Bosman et al., 2016).

\section{Support from other health professionals}

Although only mentioned in two studies, other health professionals, in addition and instead of the GP, were perceived as sources of support and guidance to facilitate discontinuation (Nygaard et al., 2015; Bosman et al., 2016). Specific professionals included mental health assistants, psychiatrists or psychologists.

\section{Sensitivity analysis}

Four studies had poorer assessments in any CASP domain compared to the other eighteen studies include in the thematic analysis of patient perspectives (Karp, 1993; McKinney and Greenfield, 2010; Nygaard et al., 2015; Weaver, 2015). The exclusion of two of these studies from the analysis, including one with the poorest assessment of study quality, did not result in any changes to our findings (McKinney and Greenfield, 2010; Weaver, 2015). However, the exclusion of the remaining two studies resulted in changes to our findings, which only affected subthemes of barriers to discontinuation (Karp, 1993; Nygaard et al., 2015). The exclusion of one study resulted in the loss of physical dependence from the subtheme "dependence" 
(Karp, 1993). The exclusion of a second study, which concerned pregnant women only, resulted in the loss of fear of effect of discontinuation on fetus from the subtheme "fear - miscelleaneous" (Nygaard et al., 2015). The exclusion of one study concerning patients who had current or recent experience of treatment for illicit drug use, did not affect our findings (Holt, 2007).

\section{DISCUSSION}

\section{Principal findings}

We identified 22 relevant studies and were able to perform a thematic synthesis of 20 primary studies reporting a patient perspective. There were insufficient data to perform a thematic synthesis for a health professional perspective.

The thematic synthesis of patient perspectives yielded nine themes and 49 subthemes. In summary, patients' psychological ability, enhanced by effective coping strategies (e.g. mindfulness based cognitive therapy) and stable life circumstances, facilitated antidepressant discontinuation. A positive perception of antidepressant treatment was linked with continuing treatment, whilst experiencing unacceptable side effects was linked with discontinuing treatment. The most common fear preventing patients from discontinuing antidepressants was relapse of their depression, while fear of addiction facilitated discontinuation. A patient's self-identity (of being 'healthy' or one's 'true self') could influence whether they saw discontinuation as a valid option. For the most part, patients reported doctors had a vital role in their decision to continue or discontinue antidepressants. There was an expectation that doctors would initiate any discussion on discontinuation, and patients were likely to follow their doctor's recommendation to continue or discontinue treatment. Other reasons for continuing treatment included a perception that depression was a long-term condition, requiring long-term treatment, or had a biochemical cause. Finally, adequate information about discontinuation, and the support of significant others, or health professionals besides their doctor, could facilitate patients discontinuing their antidepressant treatment. 


\section{Strengths of the study}

As far as we are aware, this is the first qualitative evidence synthesis specifically addressing barriers and facilitators to discontinuing long term antidepressant use.

We searched a number of databases to find relevant studies. This included a search of grey literature, which yielded four studies that would otherwise not have been located. In addition, we used supplemental searches, shown to be essential in locating further high-quality references (Papaioannou et., 2010), which yielded one study that would otherwise not have been located. Despite our comprehensive strategy, we may have missed articles as qualitative work is not as well indexed in comparison to quantitative studies e.g. RCTs (Booth, 2016).

Our use of thematic synthesis was appropriate given our research question, aim to inform intervention design, and timeframe (Booth et al., 2016). Furthermore, we included 20 studies in the thematic synthesis, which exceeds the suggestion of a minimum 12 studies for a robust analysis (Booth et al., 2016).

We provided direct quotations from included studies to ground our interpretations in the original studies (Finfgeld-Connett et al., 2010), and considered dissonance cases within our themes (Booth et al., 2015). Using this approach, we gained deeper insights into barriers and facilitators of discontinuing antidepressants than from reading any of the included studies in isolation. Nevertheless, the process of synthesising qualitative studies is inherently interpretive. Our synthesis is one possible interpretation of the data. It is possible that another research team may generate another interpretation of this set of studies.

\section{Limitations}

Due to time constraints we did deviate from our protocol. Instead of two researchers independently performing line by line coding and generating analytical themes, coding and development of subthemes and themes was performed by one researcher and further developed through discussion 
between two researchers. This method has been used in other published qualitative evidence syntheses.

\section{Comparisons with other studies}

Our initial scoping search found one qualitative evidence synthesis specifically relating to antidepressant discontinuation (Malpass et al., 2009). This meta-ethnography of patients' experiences of antidepressants, included 16 papers, five of which were included in our review (Karp, 1993; Knudsen et al., 2002; Pollack and Grime, 2002; Verbeek-Heida and Mathot, 2006; Holt, 2007). In relation to the decision to stop antidepressants, Malpass et al., identified six issues included in our study, namely: side effects, fear of addiction, ineffective or latency period of drug, authenticity, feeling better, assert control/patient agency, and psychological dependence, that were synonymous to sub-themes in our study (Malpass et al., 2009). In addition, a content analysis of patient barriers and enablers to deprescribing any long term medication included 21 studies, two of which were included in our review (Reeve et al., 2013; Verbeek-Heida and Mathot, 2006; Leydon et al., 2007). Twenty eight of 42 subthemes identified by Reeve et al., were synonymous with subthemes identified in our study (Reeve et al., 2013). However, unlike our study, neither study identified confidence in capability to discontinue, and effective coping strategies as facilitators to stopping antidepressants, nor did they identify as a barrier the patient assumption that it is the doctor's responsibility to initiate discussion of antidepressant discontinuation.

Similar subthemes to our own were also found in a recent qualitative evidence synthesis of patient experiences and perceptions of seeking and using benzodiazepines and Z-drugs (Sirdifield et al., 2017). These subthemes included: effective coping strategies e.g. changes in cognitions; fear of relapse; lack of support from GP; positive effects of benzodiazepines on negative feelings; passive feeling of trust in GPs such that patient expected GPs would initiate a change of benzodiazepine if required. 
Our themes of psychological and physical capabilities, perception of antidepressants, the fear factor, intrinsic motivators and goals, and perceived cause of depression are in agreement with Dijkstra et al.'s social cognitive theory based decision making model of discontinuing or restarting antidepressants (Dijkstra et al., 2008).

\section{Implications for clinicians and researchers}

The purpose of this review was to identify what factors influence patients' and prescribers' decisions to continue or discontinue long-term antidepressant treatment. Our findings can be utilised by doctors in order to inform their interactions with patients prescribed antidepressants. A key barrier we identified was the patient belief that the Doctor was responsible for initiating discussions about discontinuation. In a variety of illnesses and settings, it has been found that patient preference varies and that a substantial number of patients preferred less involvement in decision making than perceived (Brom et al., 2014; Cox et al., 2007). In regard to antidepressants specifically, a study by Malpass et al. found that GPs sometimes overinvested patients with autonomy and capacity to make decisions regarding treatment (Malpass et al., 2011). These findings suggest Doctors should not assume the patient will initiate a discussion about discontinuation, and instead take the impetus themselves if they feel it is appropriate for the patient to consider discontinuation. In the absence of action by the Doctor the status quo will be continuation of unnecessary antidepressant treatment for many patients. Furthermore, our findings that barriers to discontinuation include beliefs that depression has a biochemical cause and is a long-term condition requiring long-term treatment, have implications for the ways in which doctors introduce the conceptual basis for treatment when first prescribing antidepressants. Suggesting antidepressants correct a biochemical deficiency is likely to encourage a belief in the need for life-long use, yet the evidence-base for such a belief has not been established (Lacasse et al., 2005; Andrews et al., 2015). 
Our finding can be used by researchers wanting to develop safe and effective ways to support discontinuing long-term antidepressant use. We, as part of the REDUCE programme (Kendrick, 2016), aim to use the barriers and facilitators identified to inform the design of a partly internet based intervention aimed at helping patients discontinue antidepressants by providing round the clock support and guidance.

We were unable to perform a thematic synthesis of health professional perspectives due to a paucity of studies. Moreover, our thematic synthesis found that patients identified other health professionals besides doctors, such as mental health professionals, as potential sources of support in discontinuing antidepressants. Our research therefore highlights a need for further primary qualitative research of a health professional perspective including, but not limited to, GPs.

\section{Conclusion}

Barriers and facilitators to discontinuing antidepressant use are numerous and complex, and likely to require detailed conversations between patients and their GPs. Moreover, these conversations are more likely to happen if GPs raise the issue of discontinuation. Further research from a health professional perspective including, but not limited to GPs, is needed.

\section{Contributors}

EM and TK contributed to the study concept and design. EM, TK, SD, RR and HB contributed to acquisition of the data. All authors contributed to the analysis and interpretation of the data, and drafts of manuscripts. All the authors critically reviewed the manuscript for publication. TK is the study guarantor.

\section{Role of funding source}

This report is independent research funded by the National Institute for Health Research (Programme Grants for Applied Research, REviewing long term anti-Depressant Use by Careful monitoring in Everyday practice (REDUCE) programme - Ref RP-PG-1214-20004).

The funder had no role in the study design; the collection, analysis and interpretation of data; the writing of the report; or the decision to submit the article for publication.

The views expressed in this publication are those of the authors and not necessarily those of the NHS, the National Institute for Health Research or the Department of Health. 


\section{Acknowledgements}

The meaning of our findings were discussed with our Patient and Public Involvement (PPI) colleagues, who are intimately involved in the REDUCE programme to develop an interactive programme to help support patient withdrawal from antidepressants.

\section{Data sharing}

Additional data may be obtained on request to the corresponding author.

\section{Ethics approval}

Ethics approval was not required for this study

\section{Transparency statement}

The manuscript's guarantor affirms that the manuscript is an honest, accurate, and transparent account of the study being reported; that no important aspects of the study have been omitted; and that any discrepancies from the study as originally planned (and registered) have been explained. 


\section{REFERENCES}

Ambresin, G., Palmer, V., Densley, K., Dowrick, C., Gilchrist, G., Gunn, J.M., 2015. What factors influence long-term antidepressant use in primary care? Findings from the Australian diamond cohort study. J. Affect. Disord. 176, 125-132 doi: /10.1016/j.jad.2015.01.055

Anderson, K., Stowasser, D., Freeman, C., Scott, I., 2014. Prescriber barriers and enablers to minimising potentially inappropriate medications in adults: a systematic review and thematic synthesis. BMJ Open 4:e006544 doi:10.1136/bmjopen-2014-006544

Andrews, P.W., Bharwani, A., Lee, K.R., Fox, M., Thomson, J.A., 2015. Is serotonin an upper or a downer? The evolution of the serotonergic system and its role in depression and the antidepressant response. Neurosci. Biobehav. Rev.51,164-88.

Aselton, P., 2010. The Lived Experience of College Students Who have Been Medicated with Antidepressants [PhD Thesis]: University of Massachusetts - Amherst.

http://scholarworks.umass.edu/open_access_dissertations/235

Bayliss, P., Holttum, S., 2015. Experiences of antidepressant medication and cognitivebehavioural therapy for depression: a grounded theory study. Psychology and Psychotherapy. 88, 317-34.

Booth, A., Carroll, C., Ilott, I., Lan Low, L., Cooper, K., 2015. Desperately seeking dissonance: identifying the disconfirming case in qualitative evidence synthesis. Qualitative Health Research 23, 126-41.

Booth, A., 2016. Searching for qualitative research for inclusion in systematic reviews: a structured methodological review. Syst. Rev. May; 4, 74. doi: 10.1186/s13643-016-0249-x. Review

Booth, A., Noyes, J., Flemming, K., Gerhardus, A., Wahlster, P., Van Der Wilt, G.J., Mozygemba, K., Refolo, P., Sacchini, D., Tummers, M., Rehfuess, E., 2016. Guidance on choosing qualitative evidence synthesis methods for use in health technology assessments of complex interventions [Online]. Available from: http://www.integrate-hta.eu/downloads/

Bosman, R.C., Huijbregts, K.M., Verhaak, P.F.M., Ruhe, H.G., Van Marwijk, H.W.J., Van Balkom, A.J.L.M., et al., 2016. Long-term antidepressant use: A qualitative study on perspectives of patients and GPs in primary care. British Journal of General Practice 66, e708-e19.

Brom, L., Hopmans, W., Pasman, H.R., Timmermans, D.R., Widdershoven, G.A., OnwuteakaPhilipsen, B.D. 2014. Congruence between patients' preferred and perceived participation in medical decision-making: a review of the literature. BMC Med. Inform. Decis. Mak. Apr 3, 25. doi: 10.1186/1472-6947-14-25. 
Buus, N., Johannessen, H., Stage, K.B., 2012. Explanatory models of depression and treatment adherence to antidepressant medication: A qualitative interview study. International Journal of Nursing Studies 49, 1220-9.

Carroll,C., Booth, A., 2015. Quality assessment of qualitative evidence for systematic review and synthesis: Is it meaningful, and if so, how should it be performed. Research Synthesis Methods 6 , 49-54.

Coupland, C., Dhiman, P., Morriss, R., Arthur, A., Barton, G., Hippisley-Cox, J., 2011. Antidepressant use and risk of adverse outcomes in older people: population based cohort study. BMJ 343, d4551 doi: 10.1136/bmj.d4551

Cox, K., Britten, N., Hooper, R., White, P., 2007. Patients' involvement in decisions about medicines: GPs' perceptions of their preferences. British Journal of General Practice 57,777-784

Critical Appraisal Skills Programme, 2017. CASP Qualitative Checklist. [online] Available at: http://docs.wixstatic.com/ugd/dded87_25658615020e427da194a325e7773d42.pdf Accessed: 01 December 2017

Cruickshank, G., MacGillivray, S., Bruce, D., Mather, A., Matthews, K., Williams, B., 2008. Crosssectional survey of patients in receipt of long-term repeat prescriptions for antidepressant drugs in primary care. Mental Health in Family Medicine 105-9

Deci, E.L., \& Ryan, R.M., 2002. Handbook of self-determination research. University Rochester Press.

DeJean, D., Giacomini, M., Simeonov, D., Smith, A., 2016. Finding qualitative research evidence for health technology assessment. Qualitative Health Research 26, 1307-17.

Dickinson, R., Knapp, P., House, A.O., Dimri, V., Zermansky, A., Petty, D., et al. 2010. Long-term prescribing of antidepressants in the older population: A qualitative study. British Journal of General Practice 60, 257-9.

Dijkstra, A., Jaspers, M., van Zwieten, M., 2008. Psychiatric and psychological factors in patient decision making concerning antidepressant use. J. Consult. Clin. Psychol. 76, 149-57. doi: 10.1037/0022-006X.76.1.149

Eveleigh, R., 2015. Inappropriate long-term antidepressant use in primary care: a challenge to change [PhD thesis]: Radboud University Nijmegen.

Ferguson, J.M., 2001. SSRI Antidepressant Medications: Adverse Effects and Tolerability. Primary Care Companion to Journal of Clinical Psychiatry 3, 22-27. 
Finfgeld-Connett, D., 2010. Generalizability and transferability of metasynthesis research findings. Journal of Advanced Nursing 66, 246-54.doi: 10.1111/j.1365-2648.2009.05250.x

Health \& Social Care Information Centre, 2017. Prescriptions Dispensed in the Community: England, Statistics for 2006 to 2016. The Health and Social Care Information Centre. http://content.digital.nhs.uk/catalogue/PUB30014/pres-disp-com-eng-2006-16-rep.pdf

Holt, M., 2007. Agency and dependency within treatment: drug treatment clients negotiating methadone and antidepressants. Social Science \& Medicine 64, 1937-47.

Hoogen, S.R., 2006. Voice of the drug: Interpreting medicalized disempowerment in women's narratives of depression [MA thesis]: Miami University.

Iden, K.R., Hjorleifsson, S., Ruths, S., 2011. Treatment decisions on antidepressants in nursing homes: a qualitative study. Scandinavian Journal of Primary Health Care 29, 252-6.

Johnson, C.F., Williams, B., MacGillivray, S.A., Dougall, N.J., Maxwell, M., 2017. “Doing the right thing": factors influencing GP prescribing of antidepressants and prescribed doses. BMC Fam. Pract. 18,72

Johnston, O., Kumar, S., Kendall, K., Peveler, R., Gabbay, J., Kendrick, T., 2007. Qualitative study of depression management in primary care: GP and patient goals, and the value of listening. British Journal of General Practice 57, 872-9.

Karp, A., 1993. Taking antidepressant medications: resistance, trial commitment, conversion, disenchantment. Qualitative Sociology 16, 337-59.

Kendrick, T., Stuart, B., Newell, C., Geraghty, A.W.A., Moore, M., 2015. Did NICE guidelines and the Quality Outcomes Framework change GP antidepressant prescribing in England? Observational study with time trend analyses 2003-2013. J. Affect. Disord. 186, 171-77. doi:10.1016/j.jad.2015.06.052

Kendrick, A., 2016. REviewing long term anti-Depressant Use by Careful monitoring in Everyday practice (REDUCE) programme. Project number RP-PG-1214-20004. https:// europepmc.org/grantfinder/2016-2022/RP-PG-1214-20004/ [Accessed 08-02-18]

Knudsen, P., Hansen, E.H., Traulsen, J.M., Eskildsen, K., 2002. Changes in self-concept while using SSRI antidepressants. Qualitative Health Research 12, 932-44.

Lacasse, J.R., Leo, J., 2005. Serotonin and Depression: A Disconnect between the Advertisements and the Scientific Literature. PLoS Medicine. doi: 10.1371/journal.pmed.0020392

Leydon, G.M., Rodgers, L., Kendrick, T., 2007 A qualitative study of patient views on discontinuing long-term selective serotonin reuptake inhibitors. Family Practice 24, 570-5. 
Malpass, A., Shaw, A., Sharp, D., Walter, F., Feder, G., Ridd, M., Kessler, D., 2009. "Medication career" or "Moral career"? The two sides of managing antidepressants. Social Science \& Medicine 68, 154-68

Malpass, A., Kessler, D., Sharp, D., Shaw,A., 2011. 'I didn't want her to panic': unvoiced patient agendas in primary care consultations when consulting about antidepressants. British Journal of General Practice 61, e63-71. doi: 10.3399/bjgp11X556218.

McCarthy, M., 2013. Antidepressant use has doubled in rich nations in past 10 years. BMJ 347, f7261

McCrea, R.L., Sammon, C.J., Nazareth, I., Petersen, I., 2016. Initiation and duration of selective serotonin reuptake inhibitor prescribing over time: a UK cohort study. The British Journal of Psychiatry 209,421-26

McKinney, K.A., Greenfield, B.G., 2010. Self-compliance at 'Prozac campus'. Anthropology \& Medicine 17, 173-85.

McMullen, L.M., Herman, J., 2009. Women's accounts of their decision to quit taking antidepressants. Qualitative Health Research 19, 1569-79.

Moore, M., Yuen, H.M., Dunn, N., Mullee, M.A., Maskell, J., Kendrick, T., 2009. Explaining the rise in antidepressant prescribing: a descriptive study using the general practice research database. BMJ 339:b3999.

Nygaard, L., Rossen, C.B., Buus,N., 2015. Balancing Risk: A Grounded Theory Study of Pregnant Women's Decisions to (Dis)Continue Antidepressant Therapy. Issues in Mental Health Nursing 36, 485-92.

Papaioannou, D., Sutton, A., Carroll, C., Booth, A., Wong, R., 2010. Literature searching for social science systematic reviews: consideration of a range of search techniques. Health Info. Libr. J. 27, 114-22. doi: 10.1111/j.1471-1842.2009.00863.

Piek, E., Kollen, B.J., van der Meer, K., Penninx, B.W.J.H., Nolen, W.A., 2014. Maintenance Use of Antidepressants in Dutch General Practice: Non-Guideline Concordant. PLoS ONE 9, e97463. doi:10.1371/journal.pone.0097463

Pollack, K., Grime, J., 2002. Understanding Depression and its Treatment: GP, Patient and Pharmacist Perspectives. Keele University: Department of Medicines Management.

Reeve, E., To, J., Hendrix, I., Shakib, S., Roberts, M.S., Wiese, M.D., 2013. Patient barriers to and enablers of deprescribing: a systematic review. Drugs Aging 30, 793-807. 
Schofield, P., Crosland, A., Waheed, W., Aseem, S., Gask, L., Wallace, A., et al. 2011. Patients' views of antidepressants: From first experiences to becoming expert. British Journal of General Practice 61:e142-e8.

Sirdifield, C., Chipchase, S.Y., Owen, S., Siriwardena, A.N., 2017. A systematic review and metasynthesis of patients' experiences and perceptions of seeking and using benzodiazepines and Zdrugs: towards safer prescribing. Patient 10, 1-15. doi: 10.1007/s40271-016-0182-z.

Thomas, J., Harden, A., 2008. Methods for the thematic synthesis of qualitative research in systematic reviews. BMC Medical Research Methodology 8, 1-10.

Tong, A., Sainsbury, P., Craig, J., 2007. Consolidated criteria for reporting qualitative research (COREQ): a 32-item checklist for interviews and focus groups. Int. J. Qual. Health Care 9, 349-57

Vasiliadis, H,M., Latimer,E., Dionne, P.A., Préville, M., 2013. The costs associated with antidepressant use in depression and anxiety in community-living older adults. Canadian Journal of Psychiatry 58, 201-9

Verbeek-Heida, P.M., Mathot, E.F., 2006. Better safe than sorry - Why patients prefer to stop using selective serotonin reuptake inhibitor (SSRI) antidepressants but are afraid to do so: Results of a qualitative study. Chronic Illness 2, 133-42.

Weaver, A., 2015. 'Journeys through depression': patients' experiences of transformational change through mindfulness based cognitive therapy (MBCT) and antidepressant medication (ADM) [Dr of Clin Psych thesis]: University of Exeter.

Wilson, J.D., 2007. There's no meaning in chocolate: a narrative study of women's journeys beyond the disruption of depression [PhD Thesis]: Auckland University of Technology. 